\title{
Investigation of Ionospheric Plasma Flow Effects on Current Collection to Parallel Wires Using Self-Consistent Steady-State Kinetic Simulations
}

\author{
Éric Choinière* and Brian E. Gilchrist ${ }^{\dagger}$ \\ University of Michigan, Ann Arbor, Michigan 48109
}

\begin{abstract}
Multi-wire structures are needed for practical implementations of bare electrodynamic tether systems in space propulsion and radiation belt remediation applications. Using KiPS-2D, a self-consistent, steady-state kinetic solver developed at the University of Michigan, we study the sheath structure and ion current collection properties of a set of two ion-attracting cylinders immersed in a flowing plasma. The effect of plasma flow, center-tocenter spacing and orientation of the set of wires with respect to plasma flow is analyzed. Results indicate that a strong coupling exists between the cylinders' sheath structures even when their sheaths are separated spatially, whether in a stationary or flowing plasma and for both the parallel-to-flow and perpendicular-to-flow orientations. This coupling reflects strongly in the ion current collection levels observed, through unpopulated bounded ion trajectories connecting both cylinders (reducing current collection) and ion beam focusing of one cylinder onto the other (enhancing current collection). Results at wide spacings show that, when oriented parallel to plasma flow, the set of cylinders collects significantly less (by about $20 \%$ ) ion current than in a stationary plasma. Conversely, at wide spacings a set of cylinders oriented perpendicular to plasma flow collects significantly more (about 30\% more) current than in a stationary plasma. The latter enhancement is primarily attributed to the focusing of ions by each cylinder onto the other cylinder's wake-side surface.
\end{abstract}

\section{Introduction}

Future designs of complex bare electrodynamic tether systems for both propellantless space propulsion $[1$, $2]$ and radiation belt remediation through particle precipitation $[3-5]$ will require a detailed understanding of the current collection properties of conducting wires in flowing ionospheric plasmas. Multi-strand structures based on multiple parallel cylinders are being considered for two primary reasons:

- In both applications, they would provide improved survivability to collisions with micrometeroids.

- In radiation belt remediation, a multi-wire structure would allow for the formation of a large plasma sheath of equivalent size to that established using a single wire biased at a much higher bias potential. This is due to the increased capacitance of a multi-wire structure as compared to a single wire. For a given sheath size, a multi-wire structure would collect a lower amount of ion current due to the reduced bias potential, resulting in significant power savings.

In a previous paper [6], we presented simulations of two parallel cylinders immersed in a stationary plasma. Results indicated that a critical wire-to-wire spacing exists which minimizes current collection due to the existence of a set of trajectories connecting both wires (a $50 \%$ reduction was observed). Here, we study the effect of ionospheric plasma flow on the sheath structure and ion current collection to a set of two parallel conducting cylinders at various spacings. This problem is most relevant to radiation belt remediation, which uses ion-attracting potential biases. The effect of the orientation of the set of wires with respect to the

\footnotetext{
*Research Associate, Radiation Laboratory, Dept. of Electrical Engineering and Computer Science, echoinie@umich.edu

${ }^{\dagger}$ Professor, Dept. of Electrical Engineering and Computer Science and Dept. of Atmospheric, Oceanic and Space Sciences, AIAA Senior Member, gilchrst@umich.edu
} 


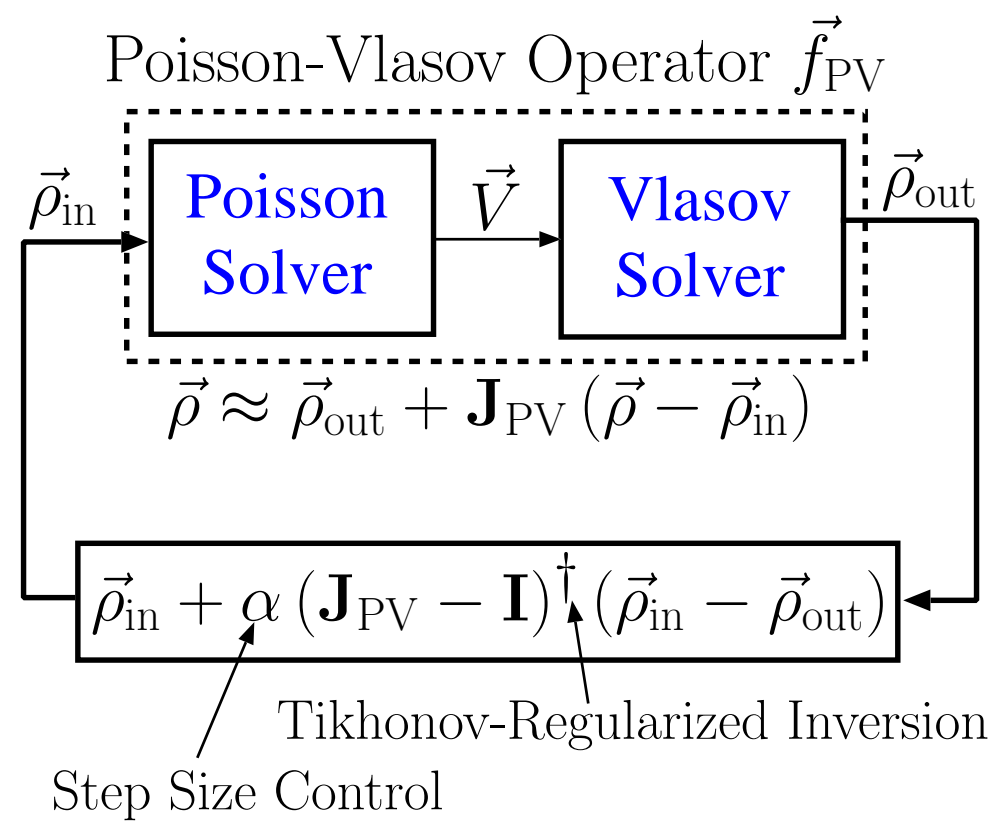

Figure 1. Progressive Tikhonov-regularized Newton iterative Poisson-Vlasov procedure. $\mathbf{J}_{P V}$ is a Jacobian matrix containing the linearized behavior of the Poisson-Vlasov operator near an "operating point" $\vec{\rho}_{\text {in }}$.

direction of plasma flow is also analyzed. All simulations are performed using KiPS-2D, a steady-state, self-consistent kinetic plasma solver developed at the University of Michigan [7].

In Section II, we present a brief overview of the kinetic model and implementation of KiPS-2D that address this class of problems, as well as an explanation of the dimensionless parameters of interest and a description of underlying assumptions.

Section III presents and analyses results of computer simulations pertaining to a set of two parallel cylinders oriented parallel and perpendicular to plasma flow, with various cylinder-to-cylinder spacings.

\section{Steady-State Self-Consistent Kinetic Model}

\section{A. Overview}

Early self-consistent analyses of the cylindrical plasma sheath by Laframboise [8] were applied to a single round cylinder immersed in a stationary plasma at low bias voltages. Other self-consistent models applicable to a single round cylinder at low bias voltages were developed which accounted for plasma flow [9-11]. In order to extend the previously available modeling capabilities to allow for large bias potentials and sheaths as well as arbitrary cylinder cross-section geometries in a flowing ionospheric plasma environment, Choinière [7] recently developed a new model along with a solver called KiPS-2D (Kinetic Plasma Solver, two-dimensional). KiPS$2 \mathrm{D}$ is a steady-state, self-consistent kinetic solver capable of simulating flowing collisionless unmagnetized plasmas in a vast region surrounding any two-dimensional conductive object. A 1-D cylindrical version of the solver, KiPS-1D, was also developed for testing and validation purposes, in addition to providing results over a much larger range of bias potentials than previously available. Validations of the KiPS solvers [7] and underlying model against previously existing models [8-10] have shown excellent agreement for round cylinders over the low voltage regimes covered by these earlier models.

The aim of the steady-state, self-consistent kinetic plasma model underlying the KiPS solvers is to provide an accurate representation of the dominant physical mechanisms involved in the formation of a steady-state sheath as well as in current collection to 2-D conductive bodies. KiPS-2D numerically solves, in a selfconsistent manner, the Poisson and Vlasov equations in the steady state over a large computational region around an arbitrarily-shaped 2-D conductive body in a collisionless, unmagnetized, flowing 2-species plasma. The computer implementation consists of successive linearizations of the nonlinear Poisson-Vlasov operator, within a Tikhonov-regularized Newton iterative process, as depicted in Fig. 1 [7]. 
Following are the main features of the KiPS model and solver:

1. It allows for the accurate representation of the complete, arbitrary velocity distribution of plasma species at all sampled locations in the computational domain.

2. It can model plasma cross flow in all speed regimes with respect to the ion thermal velocity.

3. It includes a Poisson solver based on the Finite Element Method which incorporates an outer potential boundary condition based on an asymptotic analytical result [12] for the zeroth-order circular harmonic (i.e., the azimuthal average) and assuming increasing decay rates for higher harmonics [7].

4. It provides a dynamic, adaptive, unstructured meshing strategy, allowing for the resolution of sheath asymmetries induced by the plasma flow and the support of conductive objects with arbitrary 2-D geometries;

5. It allows for the simulation of very large computational domains in order to accommodate the presheath elongation along the direction of plasma flow. The variable grid size is tailored locally to efficiently accommodate potential variations. Numerical instabilities resulting from large grid sizes are handled using a Tikhonov regularization process.

6. Its steady-state Vlasov solver features a parallel implementation based on the PVM library [13], allowing it to run on either a single host, a parallel architecture, or a scattered network of workstations based on a MPMD (Multiple Processors, Multiple Data) scheme.

Section A of the Appendix provides more detail on the implementations of both KiPS-1D and KiPS-2D.

\section{B. Normalization into Dimensionless Form}

All quantities of interest are normalized to a dimensionless form, allowing us to reduce to its bare minimum the number of parameters specifying the problem, as well as to make the simulation results shown in this paper as general as possible and therefore maximize their usefulness for system designers. Following is a description of the normalized quantities of interest:

1. Dimensionless ion and electron densities $\bar{n}_{i}$ and $\bar{n}_{e}$ are defined by $\bar{n}_{i} \equiv n_{i} / n_{0}, \bar{n}_{e} \equiv n_{e} / n_{0}$, where $n_{i}$ and $n_{e}$ are the ion and electron number densities and $n_{0}$ is the background plasma density (in $\mathrm{m}^{-3}$ ).

2. The dimensionless charge density $\bar{\rho}$ is defined in terms of the ambient density and the electron charge $e: \bar{\rho} \equiv \bar{n}_{i}-\bar{n}_{e}=\left(n_{i}-n_{e}\right) / n_{0}=\rho /\left(e n_{0}\right)$.

3. The dimensionless electric potential $\phi$ is defined by $\phi \equiv\left(V-V_{p}\right) / T_{e}$, where $V$ is the electric potential (in volts), $V_{p}$ is the plasma potential (in volts), and $T_{e}$ is the electron plasma temperature in electronvolts. The bias potential is similarly normalized according to $\phi_{0}=\left(V_{0}-V_{p}\right) / T_{e}$, where $V_{0}$ is the bias potential in volts.

4. Dimensionless coordinates $(\bar{x}, \bar{y})$ are defined by $\bar{x} \equiv x / \lambda_{D e}, \bar{y} \equiv y / \lambda_{D e}$, where $\lambda_{D e}$ is the electron Debye length in the ambient plasma. Similarly we define the dimensionless radius $\bar{r} \equiv r / \lambda_{D e}$, the dimensionless cylinder radius $\bar{r}_{0} \equiv r_{0} / \lambda_{D e}$, and the dimensionless center-to-center spacing between parallel cylinders $\overline{\Delta x} \equiv \Delta x / \lambda_{D e}$.

5. Dimensionless velocity components of ions $\left(\bar{v}_{x}, \bar{v}_{y}\right)$ are defined by $\bar{v}_{x} \equiv v_{x} / v_{t h i}$ and $\bar{v}_{y} \equiv v_{y} / v_{t h i}$, where $v_{t h i}=\sqrt{e T_{i} / m_{i}}$ is the ion thermal velocity. Similarly, the dimensionless flow velocity is given by $\bar{U} \equiv U / v_{t h i}$, where $U$ is the plasma flow velocity.

The normalizations above are consistent with the following normalization of Poisson's equation:

$$
\nabla^{2} V=-\frac{\rho}{\epsilon_{0}} \quad \Longrightarrow \quad \nabla_{n}^{2} \phi=-\bar{\rho}
$$

where $\nabla_{n}^{2}$ is the Laplacian operator applied on the normalized space coordinates $(\bar{x}, \bar{y})$. Vlasov's equation for ion trajectories can also be recast in terms of dimensionless quantities, from the usual form [14]:

$$
v_{x} \frac{\partial f}{\partial x}+v_{y} \frac{\partial f}{\partial y}-\frac{e}{m_{i}}\left(\frac{\partial V}{\partial x} \frac{\partial f}{\partial v_{x}}+\frac{\partial V}{\partial y} \frac{\partial f}{\partial v_{y}}\right)=0
$$


to the dimensionless form:

$$
\bar{v}_{x} \frac{\partial \bar{f}}{\partial \bar{x}}+\bar{v}_{y} \frac{\partial \bar{f}}{\partial \bar{y}}-\frac{T_{e}}{T_{i}}\left(\frac{\partial \phi}{\partial \bar{x}} \frac{\partial \bar{f}}{\partial \bar{v}_{x}}+\frac{\partial \phi}{\partial \bar{y}} \frac{\partial \bar{f}}{\partial \bar{v}_{y}}\right)=0,
$$

where we have introduced the ion velocity distribution function $f\left(v_{x}, v_{y}\right)$ and its dimensionless form $\bar{f}\left(\bar{v}_{x}, \bar{v}_{y}\right)$, defined as $\bar{f}\left(\bar{v}_{x}, \bar{v}_{y}\right)=\left(\frac{e T_{i}}{n_{0} m_{i}}\right) f\left(\bar{v}_{x} v_{t h i}, \bar{v}_{y} v_{t h i}\right)$. Note that the dimensionless Vlasov Equation (3) does not depend on the ion mass, but it does have a dependence on the temperature contrast between electrons and ions.

\section{Model Assumptions}

For completeness, we list here the set of assumptions relevant to the simulations presented in this paper:

1. The plasma is unmagnetized. The magnetic field force is assumed to have a negligible effect on the solution. This is appropriate as long as the in-sheath accelerated ion gyroradius is much larger than the sheath dimensions, which is certainly the case for high-voltage sheaths [7].

2. In the reference frame of the conductive cylinder, a steady state exists for the electrostatic field distributions as well as the ion and electron number density distributions. This can be confirmed $a$ posteriori by showing convergence to a self-consistent solution.

3. Cylinder end effects are negligible and the plasma and potential distributions are essentially twodimensional.

4. Away from the cylinder's perturbation, the background ion population has a drifting 2-D Maxwellian distribution given by

$$
f_{\infty}\left(v_{x}, v_{y}\right)=\frac{n_{0} m_{i}}{2 \pi e T_{i}} \exp \left\{-\frac{m_{i}}{2 e T_{i}}\left(\left(v_{x}-U\right)^{2}+v_{y}^{2}\right)\right\}
$$

where $v_{x}$ and $v_{y}$ are the two-dimensional components of the ion velocity, $n_{0}$ is the background plasma density, $m_{i}$ is the ion mass, $T_{i}$ is the ion temperature, $U$ is the plasma flow speed relative to the conductive cylinder and $e=1.6 \times 10^{-19} \mathrm{~J} / \mathrm{eV}$. The corresponding dimensionless velocity distribution is given by

$$
\bar{f}_{\infty}\left(\bar{v}_{x n}, \bar{v}_{y n}\right)=\frac{1}{2 \pi} \exp \left\{-\frac{1}{2}\left(\left(\bar{v}_{x}-\bar{U}\right)^{2}+\bar{v}_{y}^{2}\right)\right\} .
$$

5. Ions follow collisionless trajectories from the background plasma into the perturbed zone, which are guided by the self-consistent macroscopic electrostatic fields; this implies that all collision mean-freepaths involving ions are much larger than the extent of the plasma sheath in the plane perpendicular to the conductive cylinder.

6. Ions follow straight trajectories from $\bar{r}=\infty$ to the edge of the computational zone, resulting in the following incoming velocity distribution on the edge of the computational zone at $\bar{r}=\bar{r}_{M}, \theta$ :

$$
\bar{f}_{\bar{r}_{M}}\left(\bar{v}_{x}, \bar{v}_{y}\right)=\frac{1}{2 \pi} \exp \left\{-\frac{\phi_{\bar{r}_{M}, \theta}}{T_{i} / T_{e}}-\frac{1}{2}\left(\bar{v}_{x}^{2}+\bar{v}_{y}^{2}\right)-\frac{1}{2} \bar{U}^{2}+\bar{U} \bar{v}_{x} \sqrt{1+2 \frac{\phi_{\bar{r}_{M}, \theta} /\left(T_{i} / T_{e}\right)}{\bar{v}_{x}^{2}+\bar{v}_{y}^{2}}}\right\}
$$

where $\phi_{\bar{r}_{M}, \theta}$ is the dimensionless potential on the entry point at a radius $\bar{r}=\bar{r}_{M}$ and azimuth $\theta$. This assumption was also used in [15], which did not however account for flow-induced asymmetries of the potential structure.

7. Ion trapped orbits are unpopulated, i.e. all ions present in the perturbed zone can be traced back to the background plasma on collisionless trajectories.

8. Within the perturbed region, the electron density is given by the expression:

$$
\bar{n}_{e}= \begin{cases}\exp (\phi), & \phi \leq 0 \\ 1+\phi, & \phi>0\end{cases}
$$




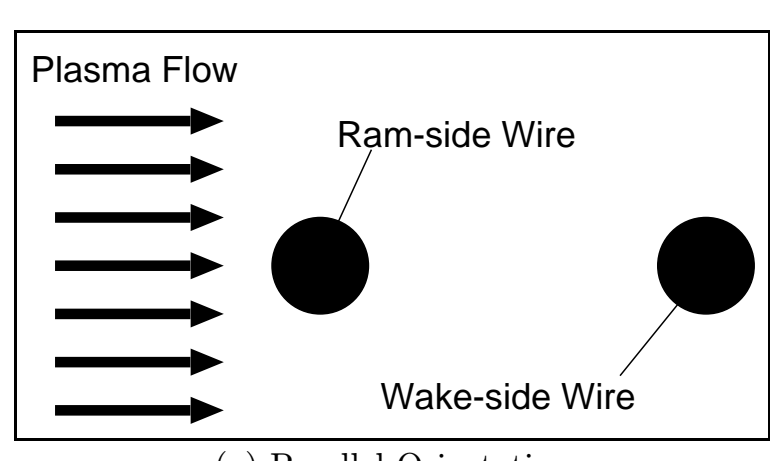

(a) Parallel Orientation

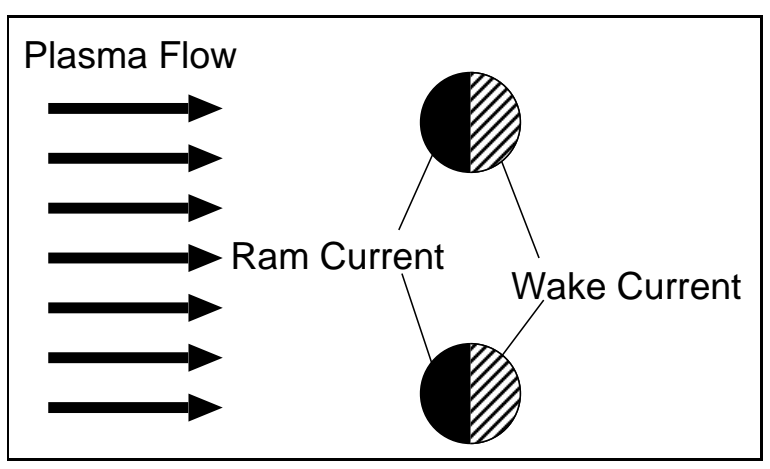

(b) Perpendicular Orientation

Figure 2. Orientations of the set of two wires with respect to plasma flow.

This corresponds rigorously to the collisionless behavior of repelled electrons at locations of negative potential $(\phi \leq 0)$, and allows for electron densities in excess of the background plasma density in shallow potential wells $(\phi>0)$ through adiabatic trapping $[16,17]$.

\section{Interference of Parallel Cylinders in Flowing Plasma: Simulation Results}

Figure 2 illustrates two orientations of the set of cylinders with respect to plasma flow that we consider in this study. In the "parallel" orientation, the wires are disposed along an axis oriented parallel to the plasma flow, whereas in the "perpendicular" orientation, they are located on an axis oriented perpendicular to the plasma flow.

\section{A. Sheath Structure}

Figures 3-5 illustrate the simulated sheath structures surrounding the two-cylinder set under three different sets of conditions:

- Stationary plasma (Figure 3).

- Plasma flowing at $80 \%$ of the ion's thermal velocity $\left(U=0.8 v_{t h i}\right)$ in a direction parallel to the orientation of the two cylinders (Figure 4).

- Plasma flowing at $80 \%$ of the ion's thermal velocity $\left(U=0.8 v_{t h i}\right)$ in a direction perpendicular to the orientation of the two cylinders (Figure 5).

All other parameters are kept constant: the cylinder radius $r_{0}=\lambda_{D e}$, the potential bias $\phi_{0}=-300$, and the temperature contrast $T_{i} / T_{e}=1$. The value used here for the flow velocity $\left(U=0.8 v_{t h i}\right)$ is representative of ionospheric conditions at an altitude of $1000 \mathrm{~km}$ with Hydrogen as the dominant species [7].

We note that only half $(\bar{y}>0)$ of the symmetric spatial distributions are shown in Figures $3-5$, in order to facilitate viewing of the internal structure details. In all simulations, plasma flow is oriented along the $\bar{x}$ axis, implying a symmetry axis coincident with the $\bar{x}$ axis. We take advantage of this symmetry by simulating only one half of the 2-D space surrounding the cylinders. In Figures 3 and 4 , the locations of the two cylinders are clearly indicated by the two distinct peaks in the potential structures, as a consequence of having placed the two cylinders on the $\bar{x}$ axis for the stationary and "parallel-to-flow" cases. However, we note that in Figure 5, which pertains to the "perpendicular-to-flow" configuration, only a single peak is visible in the potential structure. This is because the cylinders are located along the $\bar{y}$ axis, which is perpendicular to plasma flow, resulting in the second cylinder being located in the symmetric half-space not shown on the graphs.

As expected, Figures 4 and 5 indicate that strong sheath asymmetries arise from an apparently moderate flow energy as compared to the applied potential bias (the flow speed $U=0.8 v_{t h i}$ corresponds to a flow energy of $0.64 T_{i}=0.64 T_{e}$ ). Previous work on a single cylinder in a flowing plasma had indeed indicated that any plasma flow speed beyond a significant fraction of the ion's thermal velocity would result in a significant 
sheath asymmetry, and that this asymmetry would grow stronger with increasing magnitudes of the bias potential [7].

For the widest wire separation shown here $\left(\Delta x=80 \lambda_{D e}\right)$, the ion distributions around each separate wire in both flowing cases (Figures 4 and 5) resembles that which was observed around a single wire immersed in a flowing plasma [7]: a narrow density peak appears on the ram side of each wire (due to reflected ions), accompanied by a strong depression of the ion density on the wake side. However, in the case of the parallel configuration (Figure 4), one observes that the ion density incoming on the ram side of the downstream cylinder is lower than for the upstream cylinder, due to the strong deflection impressed on the incoming ion beam by the upstream cylinder. We also observe, as expected, that sheath interaction is strongest when the set of wires is oriented parallel to plasma flow.

For the closest wire separation shown here $\left(\Delta x=10 \lambda_{D e}\right)$, we find that the ion distributions is roughly independent of wire orientation with respect to the flow. Of interest is the fact that for neither orientations do we observe the usual upstream peak in ion density associated with reflected ions. In the case of a single cylinder, this local peak in upstream ion density is due to the reflection of part of the ion beam after a single orbit around the cylinder. With two cylinders, the reason for the non-occurrence of this peak is likely that the asymmetry of the potential structure around either cylinder due to the other cylinder's presence precludes the focusing of reflected ions onto a small upstream region.

\section{B. Ion Current Collection}

Figure 6a depicts the variation of total collected ion current as a function of center-to-center spacing, for all three configurations of interest (stationary case and both orientations of the flowing case). The graphs are normalized to the orbital-motion-limit current, $I_{o m l}$, which would be collected by two independent cylinders of radius $r_{0}=\lambda_{D e}$ biased at a potential $\phi_{0}=-300$ in a stationary plasma. Note that the limit of zero spacing $(\Delta x=0)$ corresponds to a single independent cylinder, which explains why the data for both orientations coincide at $\Delta x=0$. In this case the reference OML current $I_{o m l}$ is that of a single cylinder instead of two.

Figure $6 \mathrm{~b}$ provides more detail regarding the results applicable to the parallel orientation: the two separate plots pertain to the current collected by the ram-side and wake-side wires, respectively, as illustrated in Figure 2a. In the limit $\Delta x=0$, corresponding to a single cylinder as specified earlier, the data points included in the curves labeled "ram-side wire" and "wake-side wire" in Figure 2 actually represent the current collected on the ram and wake sides of the single cylinder, respectively. This is by contrast to the other data points which represent the total current collected on each of the two wires. Figure 6c applies to the perpendicular orientation, and provides plots of the current components collected on the ram side and wake side of both wires, respectively, as illustrated in Figure $2 \mathrm{~b}$.

Plasma flow is seen to significantly affect ion current collection in both the parallel and perpendicular orientations. It appears that significant effects of wire interference exist even for wire-to-wire spacings as large as $100 \lambda_{D e}$. This applies to both the stationary and flowing cases, although in different ways. Following are some specific observations relevant to each of the three cases under consideration:

Stationary Plasma. The grasph in Figure 6a shows that collected current drops from the OML value down to a minimum of about $50 \%$ of OML near a spacing of 10 Debye length. The current drop is primarily due to the existence of unpopulated trajectories connecting both cylinders [7]. It turns out that the number of such trajectories is maximized when the cylinders are spaced 10 Debye lengths apart, which is why current collection is at a minimum at this particular spacing. Current collection is then seen to rise gradually, reaching about $82 \%$ at a spacing of 100 Debye lengths. The behavior of the curve indicates that the current will rise asymptotically to $100 \%$ of OML.

Flowing Plasma, Parallel Orientation. The total current collected on both wires, as shown in Figure 6a, exhibits a few oscillations before stabilizing onto what appears to be a smooth asymptotic rise. Further cues into the mechanisms responsible for these variations are obtained by looking at the two current components shown in Figure 6b:

- The current collected on the ram-side cylinder (labeled Ram-side Wire in the legend) is just above $50 \%$ of OML for two cylinders barely touching $\left(\Delta x=2 \lambda_{D e}\right)$. This is of course because of the mutual shadowing effect caused by the near proximity of the two cylinders. Just like in the stationary case, the multiple unpopulated trajectories connecting both cylinders are responsible for the significant reduction in collected current as compared to OML. Further separation of the 
wires reduces current collection even more, as is also the case in a stationary plasma, because the surface area on each cylinder that is connected to the other cylinder trough unpopulated trajectories initially increases with spacing; the minimum is achieved at a spacing $\Delta x=8 \lambda_{D e}$, a little closer than in the stationary case. Eventually current collection ramps up and seems to stabilize at around $75 \%$ of OML, although there is an indication that this value will go down for spacings beyond the maximum tested here $\left(100 \lambda_{D e}\right)$. The fact that the result at the farthest spacing has not resumed to that obtained for a single cylinder $(\Delta x=0)$ is an indication that even at such large spacings there are a significant number of trajectories connecting both cylinders.

- The current collected on the wake-side cylinder (labeled Wake-side Wire in the legend) is about $62 \%$ of OML for two cylinders barely touching $\left(\Delta x=2 \lambda_{D e}\right)$. The peak current values observed at spacings $\Delta x=4 \lambda_{D e}$ and $\Delta x=20 \lambda_{D e}$ are very interesting. They are interpreted as being the result of a focusing effect of the ram wire which basically acts as a lens deflecting the ion beam toward the wake-side wire. According to this interpretation, the first peak would be the result of a single deflection due to the ram-side wire, causing deflected ions to hit the wake-side cylinder on its ram side. The second peak would occur at a spacing such that the initially deflected ions would not hit the wake-side cylinder directly, but instead would be deflected once more, this time by the potential structure of the wake-side cylinder, before hitting it. Beyond a spacing of $\Delta x=40 \lambda_{D e}$, the wake-side wire current rises steadily and appears to approach an asymptotic behavior that should stabilize at a somewhat lower level than the current collected on the ram-side wire, due to the combined effects of shadowing and plasma flow.

Flowing Plasma, Perpendicular Orientation. The oscillations and stabilization of the total collected ion current at a value about $8 \%$ above OML are best explained by separately considering the current components graphed in Figure 6c, that is, the current collected on the ram side of both cylinders ("Ram Current") and that collected on the wake side of both cylinders ("Wake Current").

- The ram current behaves very similarly to the current collected in a stationary plasma. It goes down to a minimum around a spacing $\Delta x=10 \lambda_{D e}$, and then goes back up, peaks at a spacing $\Delta x=38 \lambda_{D e}$, before finally stabilizing around $93 \%$ of OML. We note that this level is very close to the current obtained for a single cylinder (in the same plasma flow), which is about $96 \%$ of OML. This similarity indicates that the ram current collected on one cylinder becomes mostly independent of the presence of the other cylinder at large spacings in a flowing plasma, i.e. not very many bounded trajectories exist between the ram-side surfaces of both cylinders due to the plasma flow, contrary to the stationary case.

- The wake current ramps up quickly as the cylinders separate, with a local peak at a spacing of $10 \lambda_{D e}$, followed by a few oscillations and finally a stabilization at about $121 \%$ of OML; clearly, the wake current enhancement is responsible for the total current exceeding OML. A more detailed analysis of the simulation results will be necessary to explain the oscillations as a function of spacing. However, the fairly high value (121\%) of what appears to be an asymptotic value of the wake current is believed to be attributable to the focusing of the ion beam by one cylinder onto the other cylinder. That is, some of the incoming $\mathrm{x}$-directed ions are being deflected by one of the cylinder, onto a trajectory that ends on the wake-side surface of the other cylinder, thereby boosting the wake-side current. This enhancement of the wake-side current can exist even for very large wire-to-wire spacings, as long as no collisions are involved.

\section{Conclusion}

Using KiPS-2D, a steady-state self-consistent kinetic solver, we simulated two parallel cylinders at various spacings and orientations in a flowing ionospheric plasma, in order to investigate the effect of ion flow and cylinder interference on sheath structure and ion current collection. Strong sheath asymmetries were observed around each cylinder even at large cylinder spacings, due to the effects that each of the cylinders' sheath structure has on ion trajectories traveling through the other cylinder's sheath.

Results show that, when oriented parallel to the flow, the set of cylinders collects less ion current than in a stationary plasma for wide spacings. Conversely, the perpendicular orientation yields a much stronger current collection which even exceeds the orbital-motion limit; this strong enhancement is attributed to the focusing of ions by each cylinder onto the wake-side surface of the other cylinder. 
Our analysis indicates that each cylinders' current collection level is highly dependent on the presence of the other cylinder, even for large cylinder-to-cylinder spacings. This long-range coupling is not primarily attributable to the electric fields, since for large wire-to-wire spacings separate sheaths form around each cylinder. It is mostly due to the effect each cylinder has on the ion trajectories ending on the other cylinder, via two primary mechanisms:

1. Some bounded trajectories connect both cylinders and are not populated, causing a reduction of the ion current collection to both cylinders. This effect is observed both with a stationary and a flowing plasma.

2. In the case of a flowing plasma, one cylinder can act as a lens to focus the incoming ion beam onto the other cylinder, thereby enhancing current collection on the other cylinder. Such a focusing effect is believed to occur in the parallel orientation, where the ram-side cylinder focuses incoming ions onto the wake-side cylinder, as well as in the perpendicular orientation, where both cylinders focus ions toward the wake side of each other.

Using our simulations results, a thorough analysis based on ion trajectories will be required in order to confirm the mechanisms believed to be responsible for the observed fluctuations of the various current components. Future vacuum chamber experiments are also needed to validate the ion current collection predictions obtained here.

\section{Appendix}

\section{A. KiPS-1D and KiPS-2D: Implementations Compared}

Table 1 compares various aspects of the computer implementation of the 1-D and 2-D solvers. The primary difference between both implementations is that KiPS-1D runs on a single CPU, whereas KiPS-2D has a parallel implementation. While the 1-D implementation of the Vlasov solver does not require trajectory tracking, KiPS-2D's Vlasov solver requires tracking of several thousand particle trajectories per density sample at any given iteration, which typically totals above ten million trajectories per iteration. This can only be accomplished in a reasonable time using some form of parallelism. The 2-D version of the Vlasov solver was thus implemented in Fortran 90 using an MPMD ${ }^{\text {a }}$ parallel processing scheme based on the Parallel Virtual Machine library [13]. Since the Vlasov solver consists of computing a fixed number of plasma densities throughout the computational domain based on a given potential profile, it can easily be divided into as many independent sub-tasks. These sub-tasks are distributed to the slave nodes on a parallel platform in what constitutes a coarse-grained parallel algorithm. A dynamically-configured pool of about 100 Sun Blade $1000 / 1500$ workstations, each acting as a slave node as part of the Vlasov solver, are used as the parallel platform. A total of about 250 workstations are being used on an opportunistic basis, being swapped in and out of the 100-workstation slave node pool according to their availability. A workstation is removed from the slave node pool when a console user logs in, and only moved back in when no console users are logged in.

\section{Acknowledgments}

The authors would like to acknowledge the University of Michigan's Computer Aided Engineering Network for providing the computational resources necessary for this modeling effort.

\section{References}

\footnotetext{
${ }^{1}$ Sanmartín, J., Martínez-Sánchez, M., and Ahedo, E., "Bare Wire Anodes for Electrodynamic Tethers," Journal of Propulsion and Power, Vol. 9, No. 3, 1993, pp. 353-360.

${ }^{2}$ Estes, R., Lorenzini, E., Sanmartín, J., Peláez, J., Martínez-Sánchez, M., Johnson, C., and Vas, I., "Bare Tethers for Electrodynamic Spacecraft Propulsion," Journal of Spacecraft and Rockets, Vol. 37, No. 2, March-April 2000, pp. $205-211$.

${ }^{3}$ V. V. Danilov, V. V. Mirnov, and Ucer, D., "High-Voltage Space Tether for Enhanced Particle Scattering in Van Allen Belts," Fusion Technology, Vol. 35, No. 1T, January 1999, pp. 312-314.
}

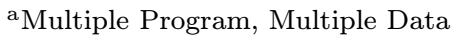




\begin{tabular}{|l|l|}
\hline \multicolumn{2}{|c|}{ IMPLEMENTATIONS COMPARED } \\
\hline \hline KiPS-1D & \multicolumn{1}{|c|}{ KiPS-2D } \\
\hline $\begin{array}{l}\text { Management of top-level iteration routine and } \\
\text { Poisson Solver under Matlab }\end{array}$ & $\begin{array}{l}\text { Serial Poisson solver, Parallel Vlasov solver } \\
\text { Poisson Solver under Matlab }\end{array}$ \\
\hline $\begin{array}{l}\text { Vlasov Solver implemented in Fortran 90 for } \\
\text { computational efficiency }\end{array}$ & $\begin{array}{l}\text { Vlasov Solver implemented in Fortran 90, runs } \\
\text { on multiple slave nodes - density samples are } \\
\text { computed by individual nodes }\end{array}$ \\
\hline Runs on a single PC & $\begin{array}{l}\text { Parallel Virtual Machine library used to harness } \\
\text { a dynamic cluster of 150 Sun Blade Workstations }\end{array}$ \\
\hline Run times from a few seconds to a few minutes & Run times from a few minutes to a few hours \\
\hline
\end{tabular}

Table 1. Comparison of the KiPS-1D and KiPS-2D implementations of the self-consistent steady-state kinetic model. Table reproduced from [6].

${ }^{4}$ V. V. Danilov, Elgin, B., Grafodatsky, O., and Mirnov, V., "High-Voltage Satellite Tethers For Active Experiments In Space," Proceedings of the 6th Spacecraft Charging Technology Conference, AFRL-VS-TR-20001578, September 2000, pp. $165-168$.

${ }^{5}$ Vasil'yev, Y. V. and V. V. Danilov, "Proposal for an Attempt to Influence the Precipitation of Charged Particles from the Earth's Radiation Belts by Means of a Satellite-Borne High-Voltage Cable System," Transaction (Doklady) of the Russian Academy of Sciences: Earth Science Sections, Vol. 345A, No. 9, October 1996, pp. 172-176.

${ }^{6}$ Choinière, É. and Gilchrist, B. E., "Poisson-Vlasov Modeling of Parallel Cylinders in Ionospheric Plasmas," AIAA Paper AIAA-2004-3496, July 2004.

${ }^{7}$ Choinière, É., Theory and Experimental Evaluation of a Consistent Steady-State Kinetic Model for 2-D Conductive Structures in Ionospheric Plasmas with Application to Bare Electrodynamic Tethers in Space, Ph.D. thesis, University of Michigan, 2004.

${ }^{8}$ Laframboise, J., Theory of Spherical and Cylindrical Langmuir Probes in a Collisionless, Maxwellian Plasma at Rest, Ph.D. thesis, University of Toronto, June 1966.

${ }^{9} \mathrm{Xu}, \mathrm{G} . \mathrm{Z}$., The Interation of a Moving Spacecraft with the Ionosphere: Current Collection and Wake Structure, Ph.D. thesis, York University, 1992.

${ }^{10}$ McMahon, J. C., The Interaction of Infinite and Finite Cylindrical Probes with a Drifting Collisionless Maxwellian Plasma, Ph.D. thesis, York University, September 2000.

${ }^{11}$ McMahon, J., Xu, G., and Laframboise, J., "The Effect of Ion Drift on the Sheath, Presheath, and Ion-Current Collection for Cylinders in a Collisionless Plasma," Physics of Plasmas, Vol. 12, No. 6, June 2005, pp. 062109-1-062109-11.

${ }^{12}$ Bernstein, I. and Rabinowitz, I., "Theory of Electrostatic Probes in a Low-Density Plasma," Physics of Fluids, Vol. 2, No. 2, March-April 1959, pp. 112-121.

${ }^{13}$ Geist, A., Beguelin, A., Dongarra, J., Jiang, W., Manchek, R., and Sunderam, V., PVM 3 User's Guide and Reference Manual, Oak Ridge National Laboratory, September 1994.

${ }^{14}$ Lieberman, M. A. and Lichtenberg, A. J., Principles of Plasma Discharges and Materials Processing, Wiley Interscience, 1994.

${ }^{15}$ Godard, R., Symmetrical Model for Cylindrical and Spherical Collectors in a Flowing Collisionless Plasma, Ph.D. thesis, York University, Canada, 1975.

${ }^{16}$ Onishi, T. and Martínez-Sánchez, M., "PIC Computation of Electron Current," 2001 International Electric Propulsion Conference - Paper IEPC-01-245, October 2001.

${ }^{17}$ Gurevich, A. V., "Distribution of Captured Particles in a Potential Well in the Absence of Collisions," Soviet Physics JETP, Vol. 26, No. 3, March 1968, pp. 575-580. 

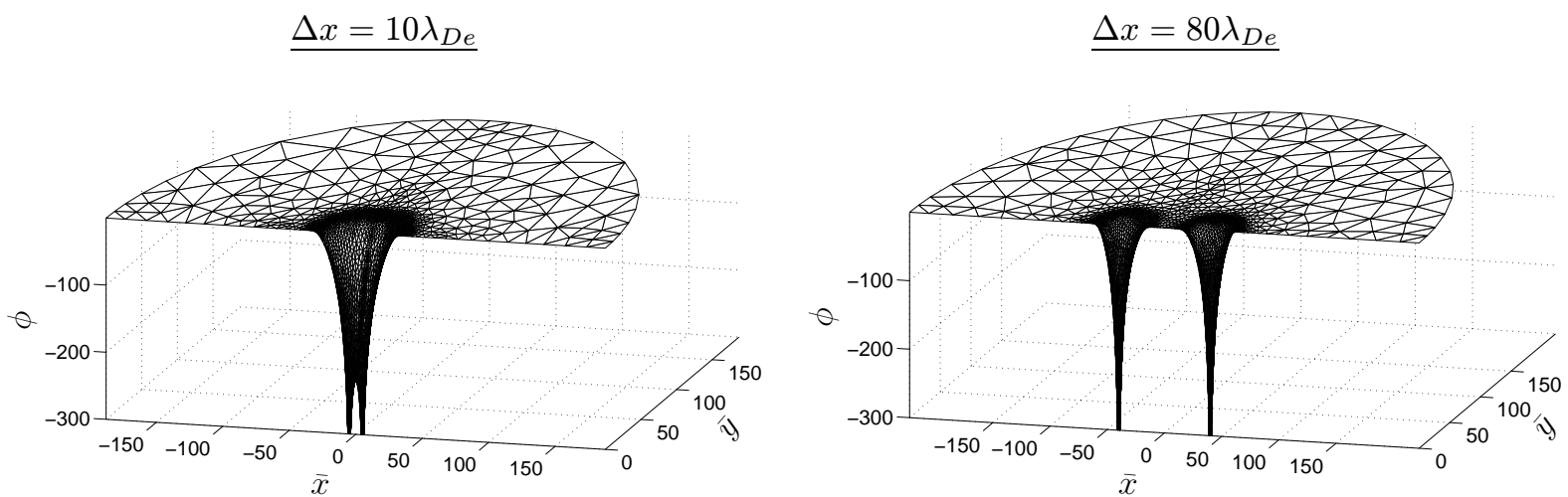

(a) Electric Potential Distributions
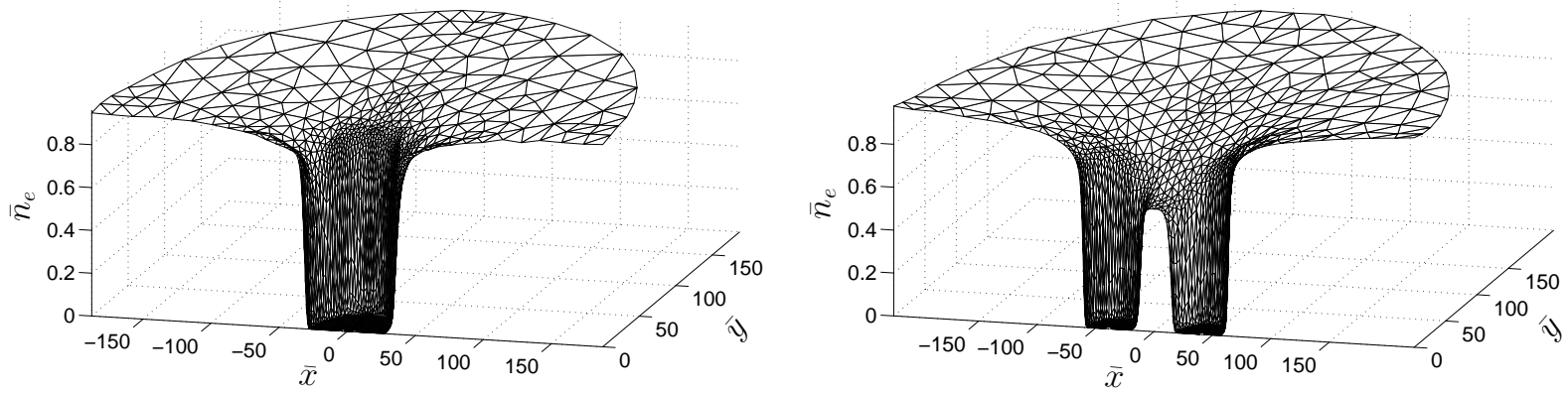

(b) Electron Number Density Distributions
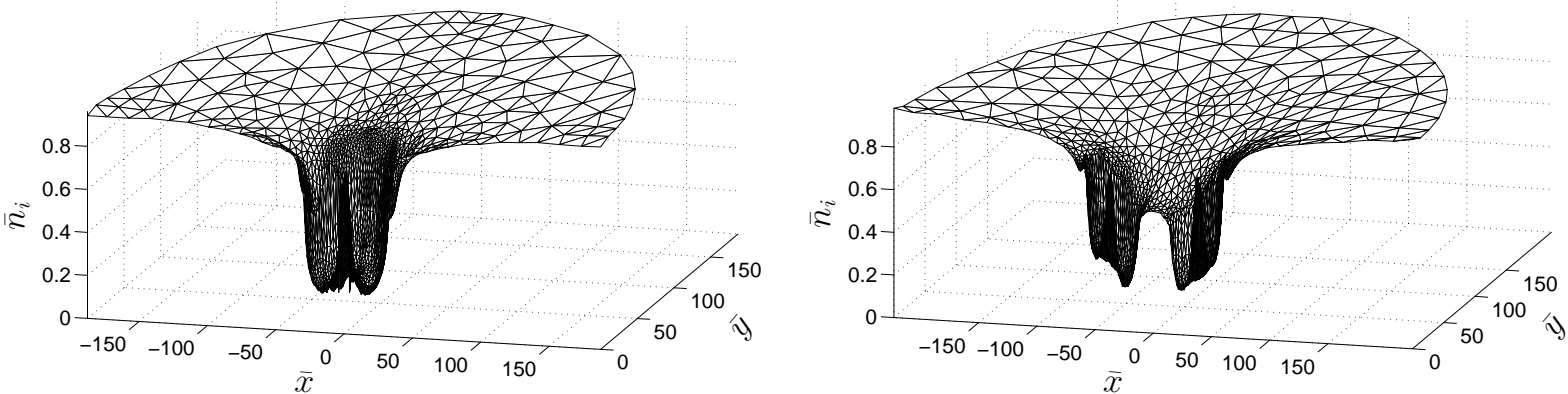

(c) Ion Number Density Distributions

Figure 3. Self-consistent solutions in a stationary plasma, with center-to-center spacings $\Delta x=10 \lambda_{D e}$ and $\Delta x=80 \lambda_{D e}$. Other parameters: $\phi_{0}=-300, r_{0}=\lambda_{D e}, T_{i} / T_{e}=1$. 

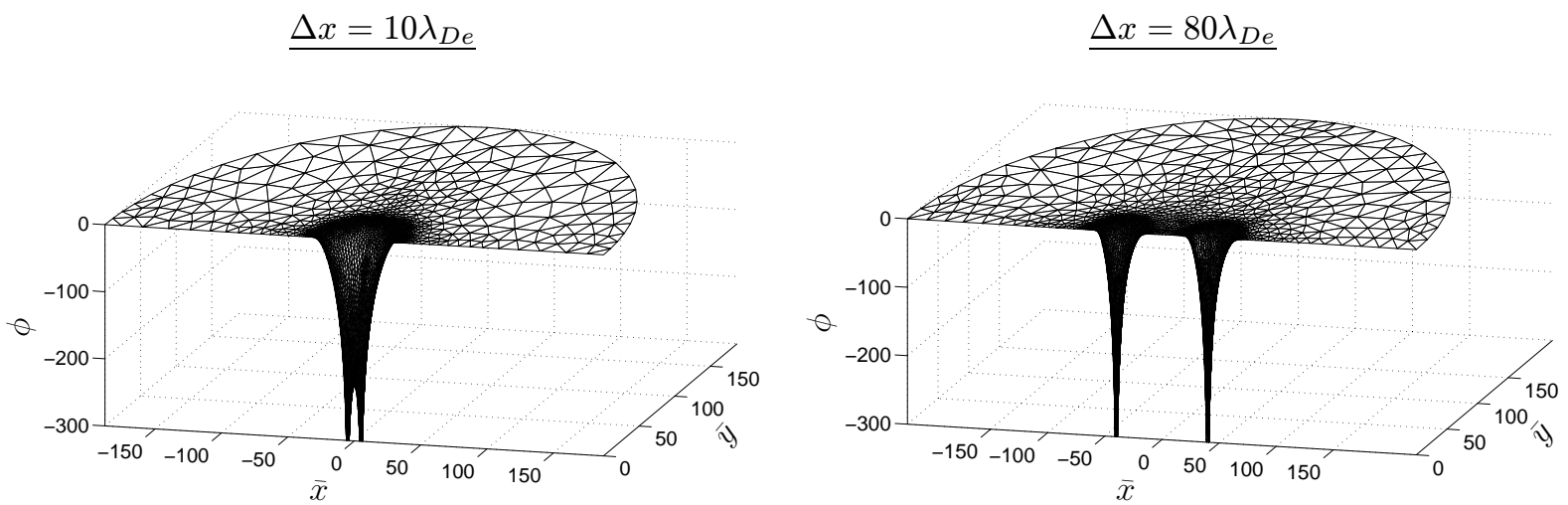

(a) Electric Potential Distributions
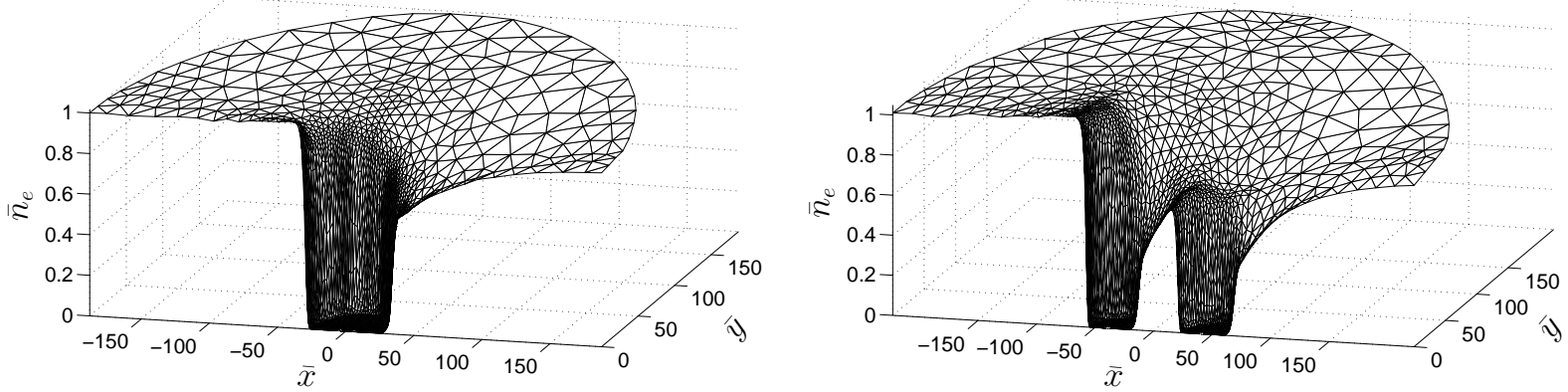

(b) Electron Number Density Distributions
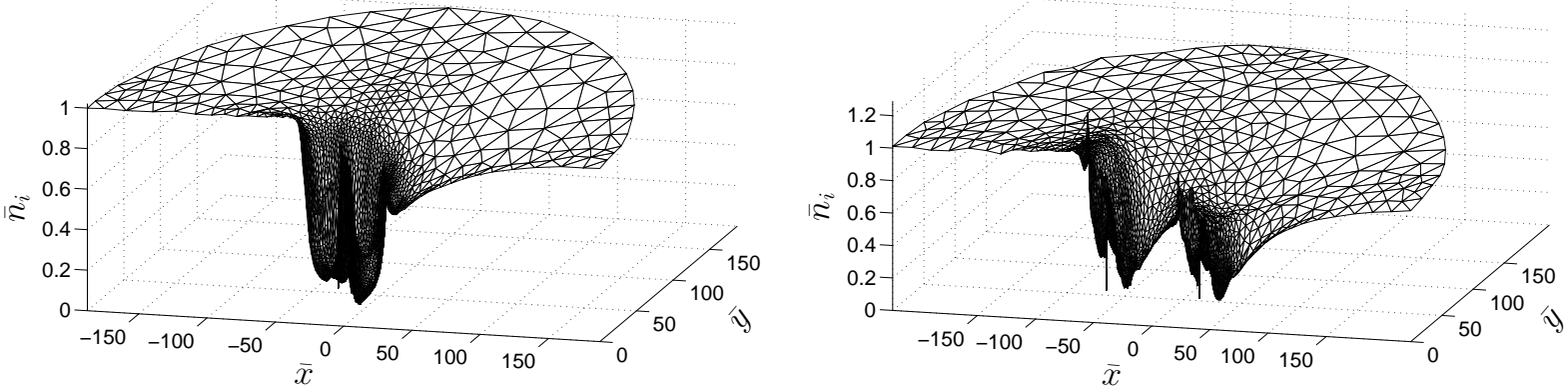

(c) Ion Number Density Distributions

Figure 4. Self-consistent solutions in a flowing plasma $\left(U=0.8 v_{t h i}\right)$ for a set of two wires oriented parallel to plasma flow, with center-to-center spacings $\Delta x=10 \lambda_{D e}$ and $\Delta x=80 \lambda_{D e}$. Other parameters: $\phi_{0}=-300$, $r_{0}=\lambda_{D e}, T_{i} / T_{e}=1$. 

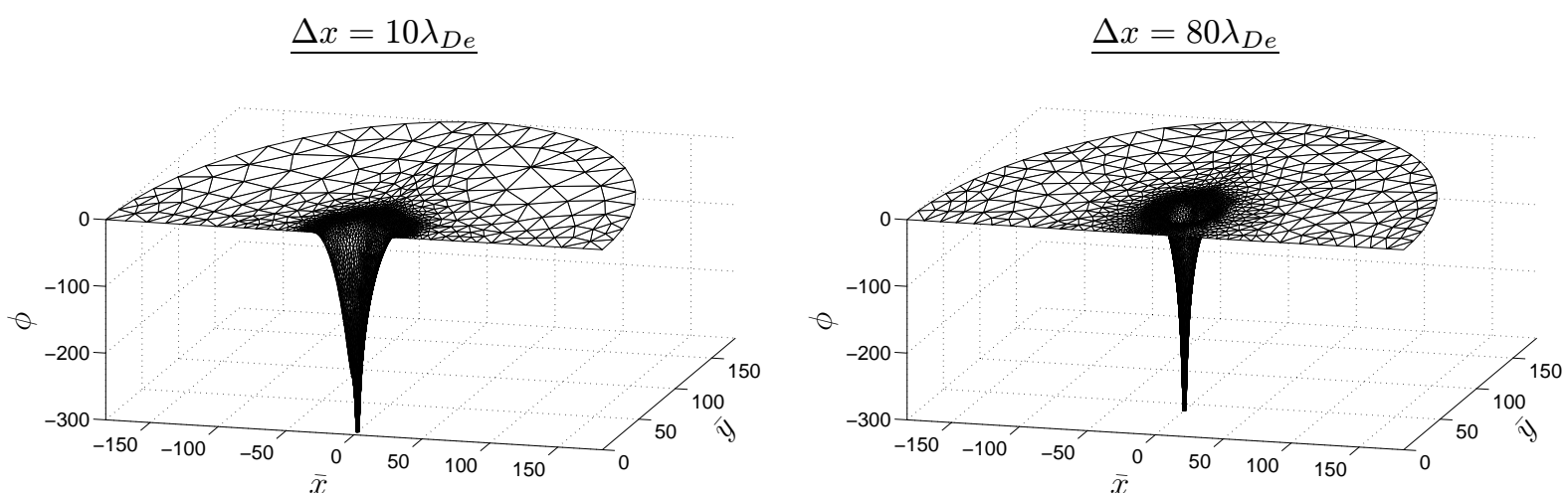

(a) Electric Potential Distributions
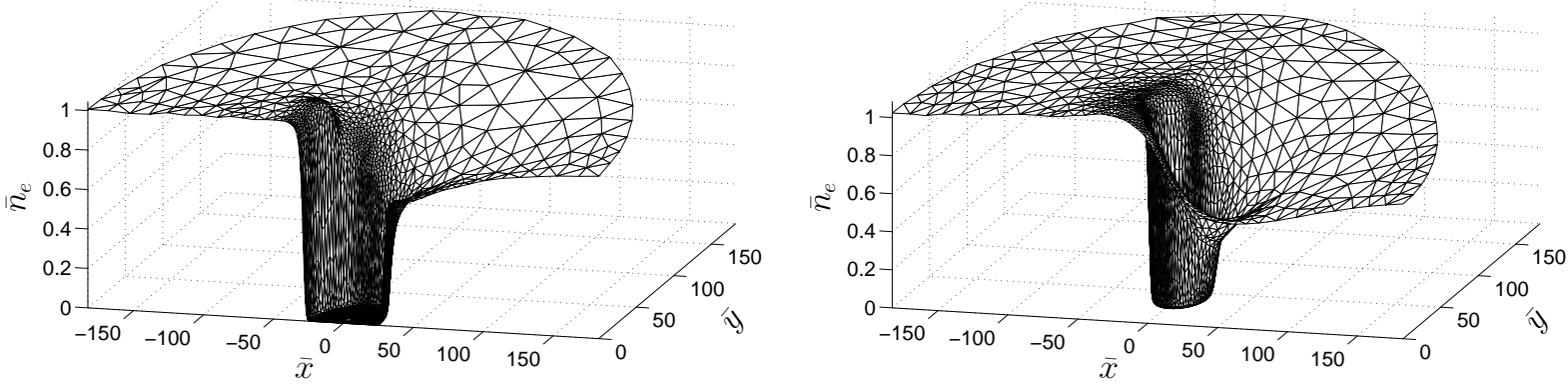

(b) Electron Number Density Distributions
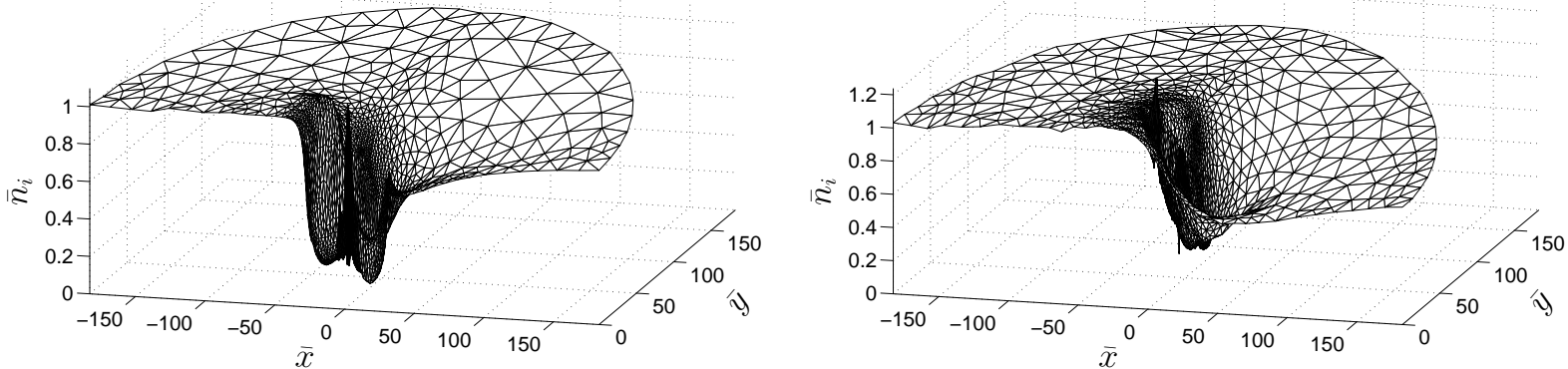

(c) Ion Number Density Distributions

Figure 5. Self-consistent solutions in a flowing plasma $\left(U=0.8 v_{t h i}\right)$ for a set of two wires oriented perpendicular to plasma flow, with center-to-center spacings $\Delta x=10 \lambda_{D e}$ and $\Delta x=80 \lambda_{D e}$. Other parameters: $\phi_{0}=-300$, $r_{0}=\lambda_{D e}, T_{i} / T_{e}=1$. 


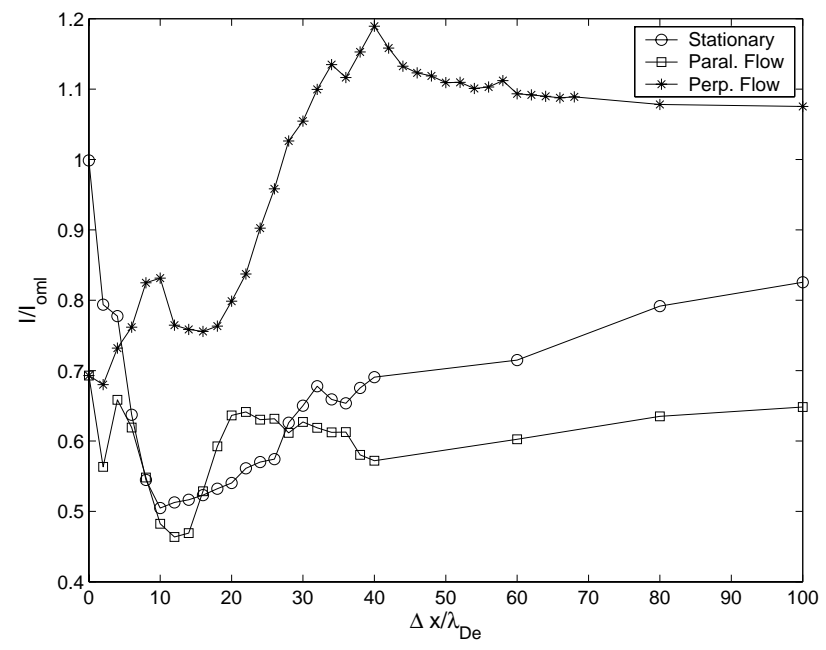

(a) Total Collected Ion Current

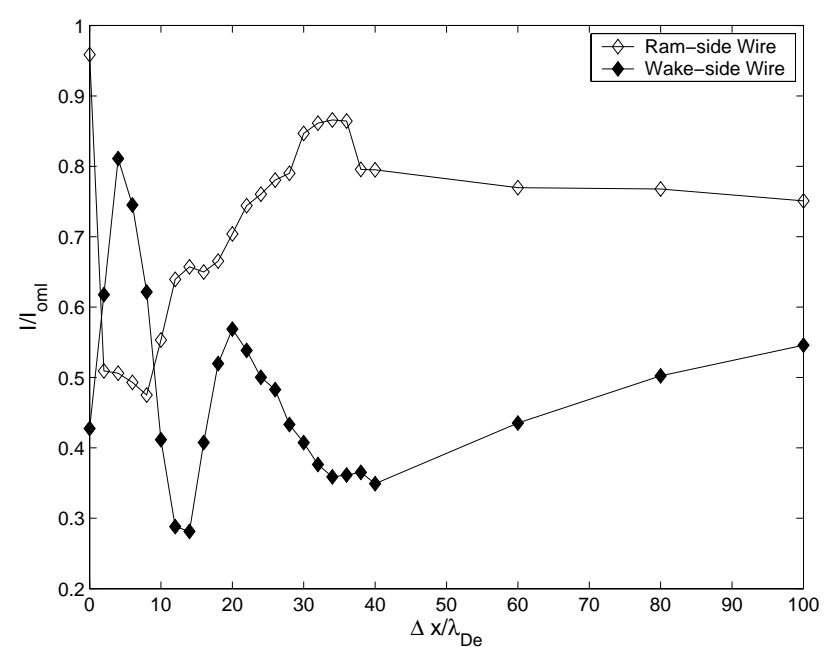

(b) Ion Current Components, Parallel Orientation

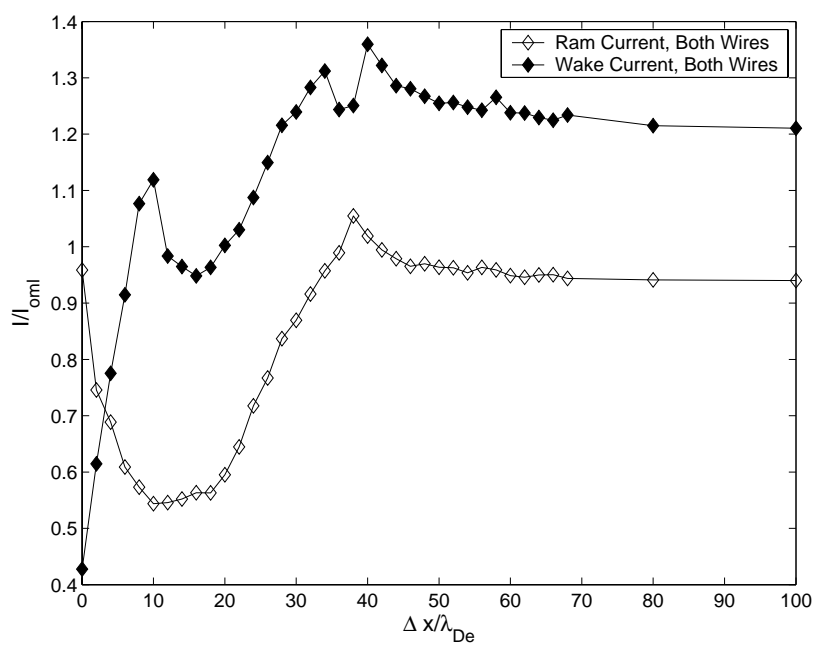

(c) Ion Current Components, Perpendicular Orientation

Figure 6. Collected ion current as a function of the center-to-center spacing $\overline{\Delta x}=\Delta x / \lambda_{D e}$ of the wires. Ion current is normalized with respect to the orbital-motion-limit value, $I_{o m l}$. (a) Total collected current in the stationary and flowing cases with results for both the parallel and perpendicular orientations of the set of wires with respect to plasma flow. (b) Current collected on the ram-side and wake-side wires, for the parallel orientation. (c) Total current collected on the ram and wake sides of both wires, for the perpendicular orientation 